Strapline: Diagnosis

\title{
Glucose dysregulation phenotypes - time to improve outcomes
}

Francesco Zaccardi and Kamlesh Khunti*

Leicester Real World Evidence Unit, Diabetes Research Centre, University of Leicester, Leicester, UK

*e-mail: kk22@leicester.ac.uk 


\section{STANDFIRST}

Detailed profiles of fluctuations in glucose obtained via continuous glucose monitoring highlight the complexity of glucose regulation and underscore that the definition of type 2 diabetes mellitus as a homogenous disease is an oversimplistic approximation for a heterogenous metabolic disorder. Whether better phenotyping would ultimately result in improved interventions and outcomes, however, remains largely unproven.

Refers to Hall, H. et al. Glucotypes reveal new patterns of glucose dysregulation. PLoS Biol. 16, e2005143 (2018).

Type 2 diabetes mellitus (T2DM) is a cardiometabolic disorder whose pathognomonic characteristic is hyperglycaemia. ${ }^{1}$ The diagnosis of T2DM is, in fact, based on the measurement of glycaemia, either during a fasting state or two hours after a challenge of oral glucose, or on the assessment of levels of $\mathrm{HbA}_{1 c}$, a parameter that is also used by clinicians to monitor glucose control as it is indicative of the mean glucose levels during the preceding 3 months.

The three criteria, however, are not always concordant as some patients could be diagnosed with T2DM according to one (for example, after glucose challenge) but not another (such as measuring levels of $\mathrm{HbA}_{1 \mathrm{c}}$ ) criterium. ${ }^{2}$ This simple observation suggests both the inappropriateness of using thresholds and categorising patients into different ranges of levels of glucose or $\mathrm{HbA}_{1 \mathrm{c}}$ and, at the same time, the oversimplification of a single condition defined as 'type 2 diabetes mellitus' despite the underlying presence of heterogeneous glucose dysregulation. ${ }^{3}$ Such heterogeneity probably reflects the different contributions of multiple pathophysiological pathways (for example, peripheral insulin resistance and $\beta$-cell failure) leading to hyperglycaemia; moreover, in epidemiological studies, different phenotypes of diabetes mellitus have also been associated with a heterogeneous risk of complications related to T2DM, mainly cardiovascular diseases. ${ }^{4}$

Over the past decade, the possibility to monitor levels of glucose for several days with continuous rather than discrete, spotted measurements, has substantially expanded our knowledge about glucose regulation and has given further insights into its heterogeneity. Examining glucose profiles has shown that excursions of glucose levels could differ among individuals with the same mean glucose level; a condition that was expected from a theoretical point of view and confirmed 
experimentally using continuous monitoring. ${ }^{5}$ As a corollary argument, it was speculated that the glucose excursions could be a risk factor for complications related to diabetes mellitus independent of mean glucose control (that is, $\mathrm{HbA}_{1 c}$ ). Such a hypothesis, popularised as 'glycaemic variability', represented a paradigm shift in the management of hyperglycaemia in diabetes mellitus, whereby healthcare professionals should not only focus on controlling mean levels of glucose $\left(\mathrm{HbA}_{1 c}\right)$ but also on glycaemic variability to reduce the risk of vascular complications. Extensive preclinical and clinical evidence further confirmed the importance of glucose variability; ${ }^{5}$ however, to date there has been no definite evidence from clinical trials of a causative role for glycaemic variability on the risk of complications related to diabetes mellitus.

The recent report by Heather Hall and colleagues ${ }^{6}$ has further shed light upon the heterogeneity of glucose regulations. In this study, using analytical methods to detail glucose excursions from continuous glucose monitoring (CGM) over several days, the glucose profiles of 57 healthy participants without a known diagnosis of T2DM were investigated. The authors classified participants according to the pattern of glucose variations as low, moderate and severe variability 'glucotypes'. Subsequently, the characteristics (age, BMI) of the patients, their metabolic profiles (fasting and post-challenge levels of glucose, peripheral insulin resistance, insulin secretion and glucose excursions following standardised meals) and cross-comparisons with established criteria of T2DM, were compared across the three glucotypes.

From a large set of almost 500,000 measurements, there are several main findings. First, some participants categorised as having no T2DM according to current diagnostic criteria have, on the contrary, glucose levels over the diagnostic thresholds for pre-diabetes or T2DM during CGM (particularly in individuals with severe glucose variability). Second, glucose excursions following a meal depend on the qualitative food composition (similar calories but varying amounts of proteins, fat, carbohydrates and fibers and, for the same food, the glucose excursions are different among participants. Third, insulin secretion, insulin resistance, and fasting and post-challenge levels of glucose correlated with glucotypes.

The pathophysiological and potential clinical implications of this study are immediately clear as firstly, glucose dysregulation is far more heterogeneous than expected and the utility of a single threshold to rigidly categorise patients in a disease status is far from being correct and 
physiologically unjustified. Secondly, the assumption of similar glucose excursions for the same amount and composition of food, which is frequently used in clinical practice to adjust insulin dose, has been disproved in this study. Thirdly, glucose profiles depend on the heterogeneous interplay between pathophysiological determinants of insulin resistance (liver, muscle) and insulin secretion ( $\beta$-cell, incretin effect). Lastly, a wider use of CGM could help identify a very early stage of glucose dysregulation in apparently 'healthy' individuals with the possibility of very early intervention potentially resulting in better longterm outcomes. As the evidence from randomised clinical trials of reducing the risk of cardiovascular outcomes and mortality following early intervention in screendetected diabetes mellitus and pre-diabetes is discordant, ${ }^{7,8}$ the phenotypes defined with the author's algorithm could result in a greater predictive value and potentially better patient outcomes. However, the widespread use of CGM should be carefully considered regarding the balance between its cost and effectiveness.

Biomedical research involved in identifying phenotypes of diseases has been extensively funded and represents one of the rising research fields of the last decade. Fuelled principally by the advancements in genetics, efforts to describe different phenotypes for medical conditions that are considered as unique nosologic entities are common in cancer, cardiovascular diseases, and diabetes mellitus medicine. The aim of such studies is to stratify the prognosis and tailor treatments, an approach commonly referred to as personalized or precision medicine. Although guidelines on precision medicine have been published to facilitate and guide researchers in translating initial observations into clinically relevant results, most of the initial promises have remained confined to early preclinical or clinical studies with unproven translated benefit for patients. As a result, for both T2DM and most other medical conditions there is very limited evidence that a model of personalized treatment approach based on detailed phenotyping would result in better longterm patient outcomes compared with the one-size-fits-all model. ${ }^{9,10}$

The pathophysiological study by Hall and colleagues represents a further step in understanding the complexity of glucose regulation and dysglycaemia in T2DM. ${ }^{6}$ However, as frequently mentioned by the authors in their article, the potential clinical implications of the results need to be ascertained. We agree and hope that their detailed investigation will not remain only an initial step in personalized medicine research; similar to other previous pathophysiological studies. The accumulating evidence of the heterogeneity of T2DM is now robust; lack of further prognostic research and of randomised clinical trials exploring the implication of personalized interventions 
suggest that researchers, institutions and public and private funders are missing the opportunity to potentially translate robust experimental results into better outcomes for patients with T2DM. 


\section{REFERENCES}

1 Zaccardi, F., Webb, D. R., Yates, T. \& Davies, M. J. Pathophysiology of type 1 and type 2 diabetes mellitus: a 90-year perspective. Postgrad Med J. 92, 63-69 (2016).

2 Mostafa, S. A. et al. The potential impact of using glycated haemoglobin as the preferred diagnostic tool for detecting Type 2 diabetes mellitus. Diabet Med. 27, 762-769 (2010).

3 Gale, E. A. Is type 2 diabetes a category error? Lancet 381, 1956-1957 (2013).

4 Ahlqvist, E. et al. Novel subgroups of adult-onset diabetes and their association with outcomes: a datadriven cluster analysis of six variables. Lancet Diabetes Endocrinol. 6, 361-369 (2018).

5 Zaccardi, F., Pitocco, D. \& Ghirlanda, G. Glycemic risk factors of diabetic vascular complications: the role of glycemic variability. Diabetes Metab Res Rev 25, 199-207 (2009).

6 Hall, H. et al. Glucotypes reveal new patterns of glucose dysregulation. PLoS Biol. 16, e2005143 (2018).

7 Gregg, E. W. \& Wing, R. Looking again at the Look AHEAD study. Lancet Diabetes Endocrinol. 5, 763-764 (2017).

8 Griffin, S. J. et al. Effect of early intensive multifactorial therapy on 5-year cardiovascular outcomes in individuals with type 2 diabetes detected by screening (ADDITION-Europe): a cluster-randomised trial. Lancet 378, 156-167 (2011).

9 Gloyn AL, Drucker DJ. Precision medicine in the management of type 2 diabetes. Lancet Diabetes Endocrinol. 2018 Apr 23. doi 10.1016/ S2213-8587(18)30052-4.

10 Psaty, B. M., Dekkers, O. M. \& Cooper, R. S. Comparison of 2 Treatment Models: Precision Medicine and Preventive Medicine. JAMA, https://dx.doi.org/10.1001/jama.2018.8377 (2018). 


\section{Acknowledgements}

F.Z. is funded and acknowledges support from an unrestricted educational grant from the National Institute for Health Research Collaboration for Leadership in Applied Health Research and Care (CLAHRC) East Midlands to the University of Leicester.

\section{Competing interests}

K.K. has acted as a consultant and speaker for Novartis, Novo Nordisk, Sanofi-Aventis, Eli Lilly and Merck Sharp \& Dohme. K.K. has received grants in support of investigator and investigator-initiated trials from AstraZeneca, Novartis, Novo Nordisk, Sanofi-Aventis, Eli Lilly, Pfizer, Boehringer Ingelheim and Merck Sharp \& Dohme.

\section{Pullquotes}

...wider use of CGM could help identify a very early stage of glucose dysregulation...

...The accumulating evidence of the heterogeneity of T2DM is now robust...

\section{Display item}

$\equiv$ : We can include one display item (Figure, Table, or Box), preferably a Figure with a legend (and ho unpublished data). However, if you feel this article is not particularly amenable to a display item, that is fine and we can use a stock image related to the article. I have included a link to a few examples of stock images that might be good]

https://www.gettyimages.co.uk/detail/photo/device-used-for-blood-glucose-test-royalty-freeimage/968121092

https://www.gettyimages.co.uk/license/905697

https://www.gettyimages.co.uk/license/916358880

Suggested stock gettyimages

981823308

896349306

816893456

Legend: Heterogeneity and continuum in the spectrum of type 2 diabetes mellitus 